\section{Late diagnosis of Henoch-Schönlein purpura following detection of jejunal ischemia on conventional endoscopy}

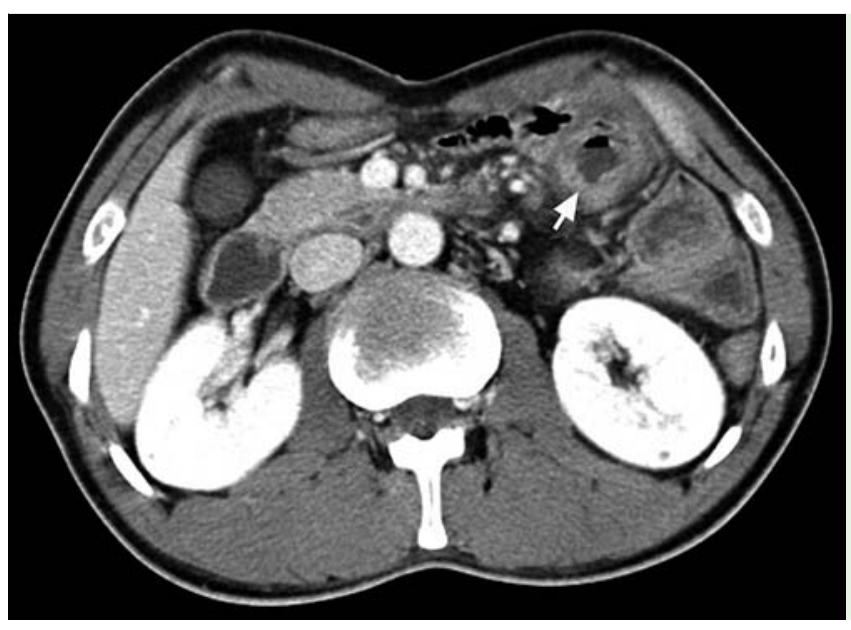

Fig. 1 Initial abdominal computed tomography (CT) scan in a 40-year-old man admitted for acute-onset, colicky abdominal pain and vomiting. There is focal concentric wall thickening and prominent mucosal enhancement around the efferent loop of the jejunum (arrow).
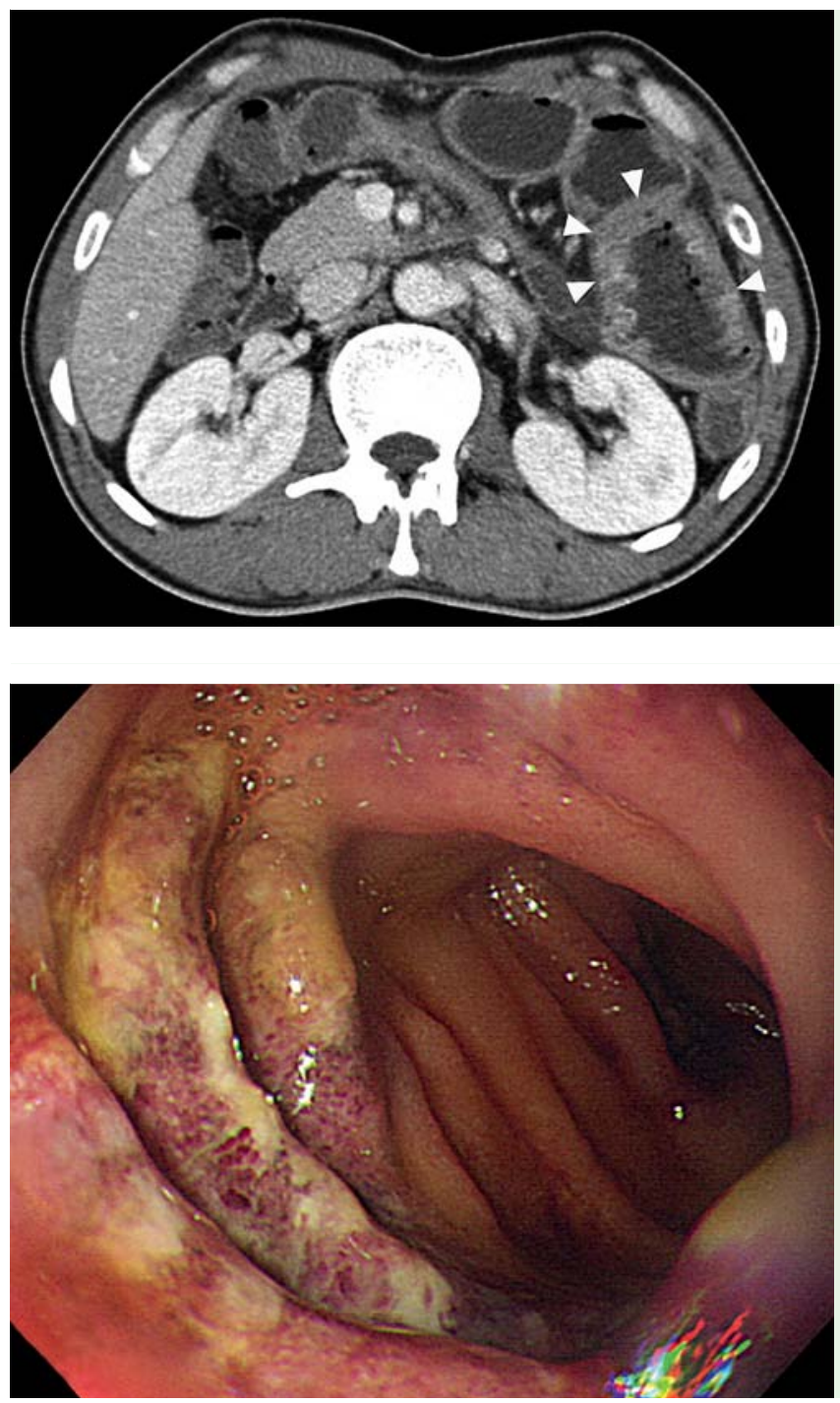

A 40-year-old man was admitted to our hospital for acute-onset, colicky abdominal pain and vomiting. He had undergone gastrojejunostomy for gastric ulcer bleeding 18 years ago. On physical examination, the patient appeared dehydrated and his abdomen was soft and diffusely tender without peritoneal signs. Laboratory investigations revealed a leukocyte count of $14890 / \mathrm{mm}^{3}$ with $91 \%$ in segment form. The initial computed tomography (CT) scan of the abdomen showed focal concentric wall thickening of the jejunum in the left upper abdomen ( $\bullet$ Fig. 1 ). Enteritis was suspected and the patient was managed with bowel rest, intravenous fluid replacement, and antibiotics. After 7 days, the patient reported slight reduction of the abdominal pain, however, the inflammatory indices were gradually deteriorating (white blood cell count: $27230 / \mathrm{mm}^{3}$ and C-reactive protein: $11.9 \mathrm{mg} / \mathrm{dL}$ ). A follow-up CT scan showed markedly aggravated wall thickening and extension of the inflamed area of the jejunum ( Fig.2). Endoscopic examination demonstrated severe segmental ischemia in the efferent loop of the jejunum ( $\bullet$ Fig. 3 ).

We decided to carry out laparotomy to resect the ischemic intestine and confirm the diagnosis, however, the patient refused surgical intervention because of the improvement in his symptoms. With the patient's informed consent, conservative management was continued. The patient's symptoms gradually improved and the inflammatory indices started to show a decrease on the twelfth day of admission. A follow-up endoscopy revealed a marked reduction in the mucosal ischemia in the efferent loop of the jejunum

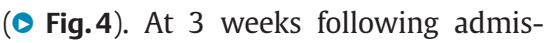
sion, the patient developed a purpuric rash, which covered his lower legs. Urinalysis revealed hematuria (20-30 red blood cells/high-power field) and marked proteinuria ( $3.5 \mathrm{~g} /$ day). Light microscopic examination of the renal biopsy samples revealed focal mesangial and endocapillary proliferation ( $\bullet$ Fig.5a) and strong positivity for immunoglobulin A (IgA, - Fig.5b) on immunofluorescent staining. A diagnosis of Henoch-Schönlein purpura (HSP) was subsequently made. The patient was discharged after 4 weeks of admission and made a good recovery.

HSP is a systemic vasculitis of unknown etiology and is thought to be related to an IgA-mediated autoimmune phenomenon [1]. Clinical diagnosis is made on the basis of the presence of physical findings such 


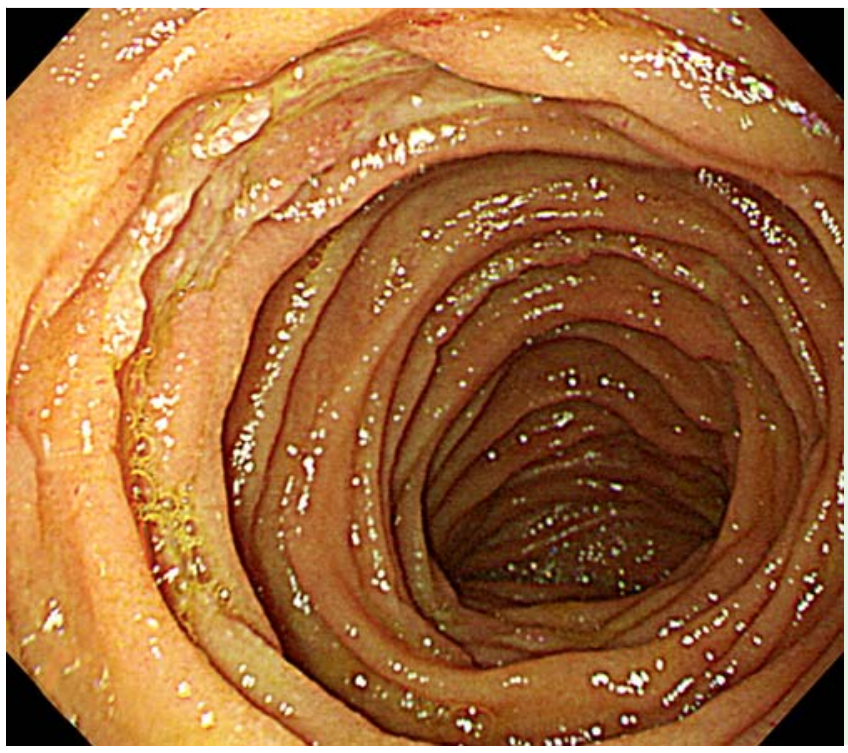
efferent loop of the
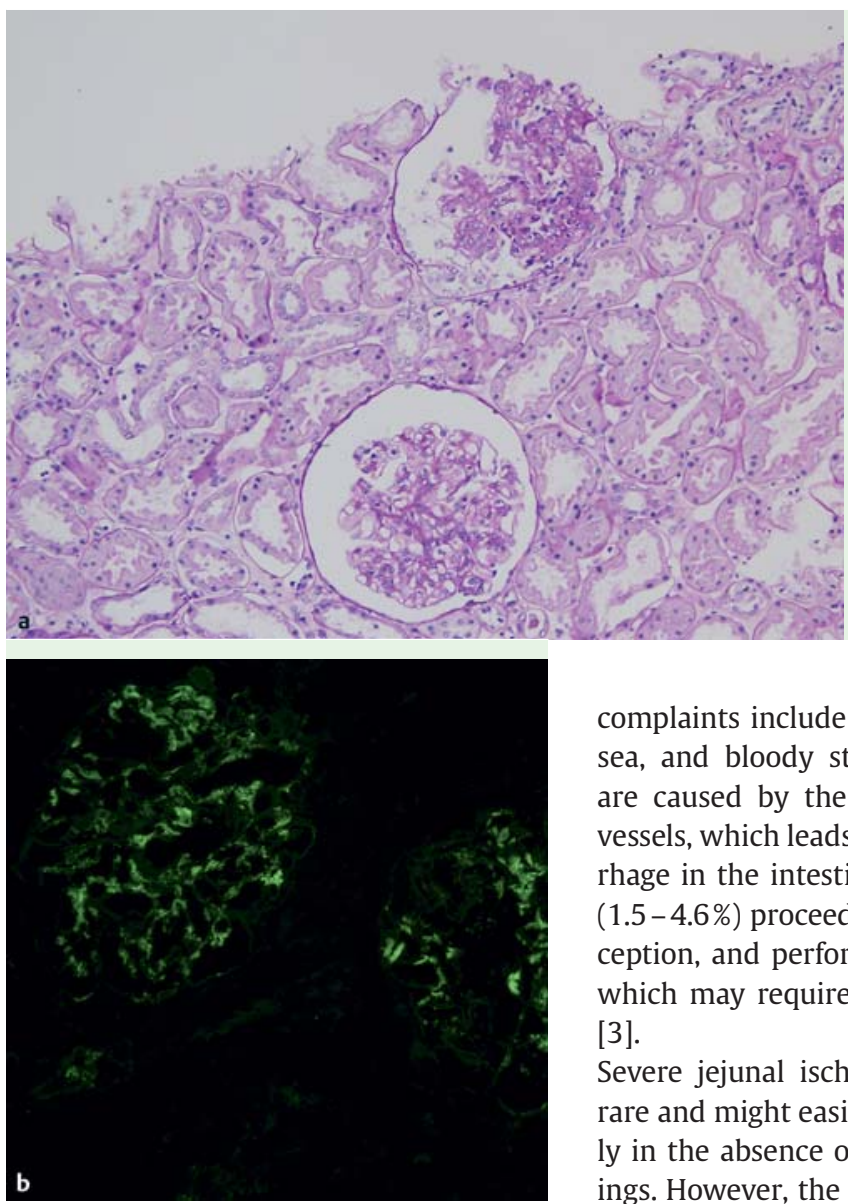
as purpuric rash, arthralgia, abdominal pain, and renal complications [2]. The gastrointestinal system is one of the most frequently involved systems in children with HSP and common gastrointestinal-related
Fig. 4 Follow-up endoscopic examination showing marked improvement of mucosal ischemia in the jejunum.

Fig. 5 Histological findings of the renal biopsy. a Focal mesangial and endocapillary proliferation was observed on light microscopic examination (periodic acid-Schiff [PAS] stain; $\times 200$ ). b Immunofluorescent studies showing strong positivity for IgA, predominantly in the glomerular mesangium $(\times 200)$.

complaints include abdominal pain, nausea, and bloody stool. These symptoms are caused by the inflammation of the vessels, which leads to edema and hemorrhage in the intestinal wall. Severe cases (1.5-4.6\%) proceed to necrosis, intussusception, and perforation of the intestine, which may require surgical intervention [3].

Severe jejunal ischemia in adult HSP is rare and might easily be missed, especially in the absence of dermatological findings. However, the upper gastrointestinal endoscopic findings that are suggestive of

paid to gastrointestinal manifestations and the decision to undertake surgical intervention should depend on the patient's symptoms rather than the laboratory results or imaging studies.

\section{Endoscopy_UCTN_Code_CCL_1AC_2AD}

\section{Competing interests: None}

\section{E. H. Kim', B. H. Park ${ }^{2}$, J. W. Lim', S. W. Jung ${ }^{1}$}

${ }^{1}$ Department of Internal Medicine, University of Ulsan College of Medicine, Ulsan University Hospital, Ulsan, Korea

${ }^{2}$ Department of Pathology, University of Ulsan College of Medicine, Ulsan University Hospital, Ulsan, Korea

\section{References}

1 Lahita RG. Influence of age on Henoch Schonlein purpura. Lancet 1997; 350: 1116- 1117

2 Allen DM, Diamond LK, Howell DA. Anaphylactoid purpura in children (SchonleinHenoch syndrome): review with a followup of the renal complications. AMA J Dis Child 1960; 99: 833-854

3 Choong CK, Beasley SW. Intra-abdominal manifestations of Henoch-Schonlein purpura. J Paediatr Child Health 1998; 34: 405-409

4 Kato S, Shibuya H, Naganuma H et al. Gastrointestinal endoscopy in Henoch-Schonlein purpura. Eur J Pediatr 1992; 151: 482 - 484

5 Banerjee B, Rashid S, Singh E et al. Endoscopic findings in Henoch-Schonlein purpura. Gastrointest Endosc 1991; 37: 569571

\section{Bibliography}

DOI http://dx.doi.org/

10.1055/s-0032-1310247

Endoscopy 2012; 44: E393-E394

(c) Georg Thieme Verlag KG

Stuttgart · New York

ISSN 0013-726X

\section{Corresponding author}

\section{S. W. Jung}

Department of Internal Medicine

University of Ulsan College of Medicine

Ulsan University Hospital

290-3 Jeonha-dong

Dong-gu

Ulsan 682-714

Korea

might be considered as a manifestation of adults with acute-onset symptoms. Therefore, special attention should be
Fax: +82-52-2507048

swjung@uuh.ulsan.kr 